The Centre of Unitary Isotopes of $J B^{*}$-Algebras

\author{
AKHLAQ A. SIDDIQUI
}

\begin{abstract}
We identify the centre of unitary isotopes of a $J B^{*}$-algebra. We show that the centres of any two unitary isotopes of a $J B^{*}$-algebra are isometrically Jordan *-isomorphic to each other. However, there need be no inclusion between centres of the two unitary isotopes.
\end{abstract}

\title{
1. BASICS
}

We begin by recalling (from [3], for instance) the following concepts of homotope and isotope of Jordan algebras.

Let $\mathcal{J}$ be a Jordan algebra, cf. [3], and $x \in \mathcal{J}$. The $x$-homotope of $\mathcal{J}$, denoted by $\mathcal{J}_{[x]}$, is the Jordan algebra consisting of the same elements and linear algebra structure as $\mathcal{J}$ but a different product, denoted by ".x", defined by

$$
a \cdot x=\{a x b\}
$$

for all $a, b$ in $\mathcal{J}_{[x]}$. By $\{p q r\}$ we will always denote the Jordan triple product of $p, q, r$ defined in the Jordan algebra $\mathcal{J}$ as below:

$$
\{p q r\}=(p \circ q) \circ r-(p \circ r) \circ q+(q \circ r) \circ p,
$$

where $\circ$ stands for the original Jordan product in $\mathcal{J}$. An element $x$ of a Jordan algebra $\mathcal{J}$ with unit $e$ is said to be invertible if there exists $x^{-1} \in \mathcal{J}$, called the inverse of $x$, such that $x \circ x^{-1}=e$ and $x^{2} \circ x^{-1}=x$. The set of all invertible elements of $\mathcal{J}$ will be denoted by $\mathcal{J}_{\text {inv }}$. In this case, $x$ acts as the unit for the homotope $\mathcal{J}_{\left[x^{-1}\right]}$ of $\mathcal{J}$.

If $\mathcal{J}$ is a unital Jordan algebra and $x \in \mathcal{J}_{\text {inv }}$ then by $x$-isotope of $\mathcal{J}$, denoted by $\mathcal{J}^{[x]}$, we mean the $x^{-1}$-homotope $\mathcal{J}_{\left[x^{-1}\right]}$ of $\mathcal{J}$. We denote the multiplication " $\cdot_{x^{-1}}$ " of $\mathcal{J}^{[x]}$ by " $\circ_{x}$ ".

The following lemma gives the invariance of the set of invertible elements in a unital Jordan algebra on passage to any of its isotopes. 
Lemma 1.1. For any invertible element a in a unital Jordan algebra $\mathcal{J}, \mathcal{J}_{\text {inv }}=\mathcal{J}_{\text {inv }}^{[a]}$.

Proof. See Lemma 1.5 of [8].

Let $\mathcal{J}$ be a Jordan algebra and let $a, b \in \mathcal{J}$. The operators $T_{b}$ and $U_{a, b}$ are defined on $\mathcal{J}$ by $T_{b}(x)=b \circ x$ and $U_{a, b}(x)=\{a x b\}$. We shall denote $U_{a, a}$ simply by $U_{a}$. The elements $a$ and $b$ are said to operator commute if $T_{a}$ commute with $T_{b}$.

Let $\mathcal{J}$ be a complex unital Banach Jordan algebra and let $x \in \mathcal{J}$. As usual, the spectrum of $x$ in $\mathcal{J}$, denoted by $\sigma_{\mathcal{J}}(x)$, is defined by

$$
\sigma_{\mathcal{J}}(x)=\{\lambda \notin \mathbb{C}: x-\lambda e \text { is not invertible in } \mathcal{J}\} .
$$

A Jordan algebra $\mathcal{J}$ with product $\circ$ is called a Banach Jordan algebra if there is a norm $\|\cdot\|$ on $\mathcal{J}$ such that $(\mathcal{J},\|\|$.$) is a$ Banach space and $\|a \circ b\| \leq\|a\|\|b\|$. If, in addition, $\mathcal{J}$ has a unit $e$ with $\|e\|=1$ then $\mathcal{J}$ is called a unital Banach Jordan algebra. In the sequel, we will only be considering unital Banach Jordan algebras; the norm closure of the Jordan subalgebra $J\left(x_{1}, \ldots, x_{r}\right)$ generated by $x_{1}, \ldots, x_{r}$ of Banach Jordan algebra $\mathcal{J}$ will be denoted by $\mathcal{J}\left(x_{1}, \ldots, x_{r}\right)$.

The following elementary properties of Banach Jordan algebras are similar to those of Banach algebras and their proofs are a fairly routine modifications of these $[1,2,7,9]$.

Lemma 1.2. Let $\mathcal{J}$ be a Banach Jordan algebra with unit e and $x_{1}, \ldots, x_{r} \in \mathcal{J}$.

(i) If $J\left(x_{1}, \ldots, x_{r}\right)$ is an associative subalgebra of $\mathcal{J}$, then $\mathcal{J}\left(x_{1}, \ldots, x_{r}\right)$ is a commutative Banach algebra.

(ii) $T_{x_{1}}$ and $U_{x_{1}, x_{2}}$ are continuous with $\left\|T_{x_{1}}\right\| \leq\left\|x_{1}\right\|$ and $\left\|U_{x_{1}, x_{2}}\right\| \leq 3\left\|x_{1}\right\|\left\|x_{2}\right\|$.

(iii) $\mathcal{J}\left(x_{1}, \ldots, x_{r}\right)$ is a closed subalgebra of $\mathcal{J}$.

(iv) If $\mathcal{J}$ is unital then $\mathcal{J}\left(e, x_{1}\right)$ is a commutative Banach algebra.

(v) If $x \in \mathcal{J}$ and $\|x\|<1$ then $e-x$ is invertible and $(e-x)^{-1}=$ $\sum_{n=0}^{\infty} x^{n} \in \mathcal{J}(e, x)$.

(vi) If $K$ is a closed Jordan subalgebra of $\mathcal{J}$ containing $e$ and $x \in K$ such that $\mathbb{C} \backslash \sigma_{\mathcal{J}}(x)$ is connected then $\sigma_{\mathcal{J}}(x)=$ $\sigma_{K}(x)$. 
We are interested in a special class of Banach Jordan algebras, called $J B^{*}$-algebras. These include all $C^{*}$-algebras as a proper subclass (see $[10,13])$.

A complex Banach Jordan algebra $\mathcal{J}$ with isometric involution * (see [6], for instance) is called a $J B^{*}$-algebra if $\left\|\left\{x x^{*} x\right\}\right\|=\|x\|^{3}$ for all $x \in \mathcal{J}$.

The class of $J B^{*}$-algebras was introduced by Kaplansky in 1976 (see [10]) around the same time when a related class called $J B$ algebras was being studied by Alfsen, Shultz and Størmer (see [1]).

A real Banach Jordan algebra $\mathcal{J}$ is called a $J B$-algebra if $\|x\|^{2}=$ $\left\|x^{2}\right\| \leq\left\|x^{2}+y^{2}\right\|$ for all $x, y \in \mathcal{J}$.

These two classes of algebras are linked as follows (see $[10,13])$.

Theorem 1.3. (a) If $\mathcal{A}$ is a $J B^{*}$-algebra then the set of self-adjoint elements of $\mathcal{A}$ is a JB-algebra.

(b) If $\mathcal{B}$ is a JB-algebra then under a suitable norm the complexification $\mathcal{C}_{\mathcal{B}}$ of $\mathcal{B}$ is a $J B^{*}$-algebra.

There is an easier subclass of these algebras. Let $\mathcal{H}$ be a complex Hilbert space and let $\mathcal{B}(\mathcal{H})$ denote the full algebra of bounded linear operators on $\mathcal{H}$.

(a) Any closed self-adjoint complex Jordan subalgebra of $\mathcal{B}(\mathcal{H})$ is called a $J C^{*}$-algebra.

(b) Any closed real Jordan subalgebra of self-adjoint operators of $\mathcal{B}(\mathcal{H})$ is called a $J C$-algebra.

Any $J B^{*}$-algebra isometrically *-isomorphic to a $J C^{*}$-algebra is also called a $J C^{*}$-algebra; similarly, any $J B$-algebra isometrically isomorphic to a $J C$-algebra is also called a $J C$-algebra.

It is easy to verify that a $J C^{*}$-algebra is a $J B^{*}$-algebra and a $J C$-algebra is a $J B$-algebra. It might be expected, conversely, that every $J B$-algebra is a $J C$-algebra (with a corresponding statement for $J B^{*}$-algebras and $J C^{*}$-algebras) but unfortunately this is not true (for details see [1]).

\section{Unitary Isotopes of a $J B^{*}$-ALgebra}

In [8], we presented a study of unitary isotopes of $J B^{*}$-algebras. In this section, we recall some facts from [8] which are needed for the sequel.

Let $\mathcal{J}$ be a $J B^{*}$-algebra. The element $u \in \mathcal{J}$ is called unitary if $u^{*}=u^{-1}$, the inverse of $u$. The set of all unitary elements of $\mathcal{J}$ 
will be denoted by $\mathcal{U}(\mathcal{J})$. If $u$ is a unitary element of $J B^{*}$-algebra $\mathcal{J}$ then the isotope $\mathcal{J}^{[u]}$ is called a unitary isotope of $\mathcal{J}$.

Theorem 2.1. Let $u$ be a unitary element of the $J B^{*}$-algebra $\mathcal{J}$. Then the isotope $\mathcal{J}^{[u]}$ is a JB*-algebra having $u$ as its unit with respect to the original norm and the involution $*_{u}$ defined by $x^{*_{u}}=$ $\left\{u x^{*} u\right\}$.

Proof. See Theorem 2.4 of [8].

Recall (from [3], for instance) that a Jordan algebra is said to be special if it is isomorphic to a Jordan subalgebra of some associative algebra. We require the following fact.

Lemma 2.2. If $\mathcal{J}$ is a special Jordan algebra and $a \in \mathcal{J}$, then $\mathcal{J}_{[a]}$ is a special Jordan algebra.

Proof. See Lemma 1.3 in [8].

Theorem 2.3. The unitary isotope of a $\mathrm{JC}^{*}$-algebra is again a $\mathrm{JC}^{*}$ algebra.

Proof. This follows from Theorem 2.1 and Lemma 2.2 (also see [8, Theorem 2.12]).

We close this section by noting following facts.

Lemma 2.4. Let $\mathcal{J}$ be a JB*-algebra with unit $e$. Then $u \in$ $\mathcal{U}(\mathcal{J}) \Longrightarrow e \in \mathcal{U}\left(\mathcal{J}^{[u]}\right)$. Moreover $\mathcal{J}^{[u]^{[e]}}=\mathcal{J}$.

Proof. See Lemma 2.7 of [8].

Next theorem establishes the invariance of unitaries on passage to unitary isotopes of a $J B^{*}$-algebra.

Theorem 2.5. For any unitary element $u$ in the $J B^{*}$-algebra $\mathcal{J}$,

$$
\mathcal{U}(\mathcal{J})=\mathcal{U}\left(\mathcal{J}^{[u]}\right) .
$$

Proof. See Theorem 2.8 of [8].

Corollary 2.6. Let $\mathcal{J}$ be a $J B^{*}$-algebra with unit $e$ and let $u, v \in$ $\mathcal{U}(\mathcal{J})$. Then

(i) $\mathcal{J}^{[u]^{[v]}}=\mathcal{J}^{[v]}$.

(ii) The relation of being unitary isotope is an equivalence relation in the class of unital $J B^{*}$-algebras.

Proof. See Corollary 2.9 of [8]. 


\section{Centre of Unitary Isotopes}

In this section, we identify the centre of unitary isotopes in terms of the centre of the original $J B^{*}$-algebra. We recall the following definition from [14].

Definition 3.1. Let $\mathcal{J}$ be a unital $J B^{*}$-algebra and let

$C(\mathcal{J})=\left\{x \in \mathcal{J}_{\text {sa }}: x\right.$ operator commutes with every y $\left.\in \mathcal{J}_{\text {sa }}\right\}$.

Then the centre of $\mathcal{J}$, denoted by $\mathcal{Z}(\mathcal{J})$, is defined by

$$
\mathcal{Z}(\mathcal{J})=C(\mathcal{J})+i C(\mathcal{J}) .
$$

Remark 3.2. It is known from [14] that $\mathcal{Z}(\mathcal{J})$ is a $C^{*}$-algebra, and if $\mathcal{J}$ is a $J C^{*}$-algebra with $\mathcal{J} \subseteq \mathcal{B}(\mathcal{H})$ for some Hilbert space $\mathcal{H}$ then

$$
\mathcal{Z}(\mathcal{J})=\{x \in \mathcal{J}: x y=y x \quad \forall y \in \mathcal{J}\} .
$$

To investigate further properties of the centre we need the following lemma.

Lemma 3.3. Let $\mathcal{J}$ be a $J B^{*}$-algebra and let $x \in \mathcal{Z}(\mathcal{J})$. Then for all $y \in \mathcal{J}$,

(i) $T_{x} T_{y}=T_{y} T_{x}$;

(ii) $T_{x} U_{y}=U_{y} T_{x}$

(iii) $U_{x} U_{y}=U_{y} U_{x}$

(iv) if $u \in \mathcal{J}$ is unitary then $\left(x \circ u^{*}\right) \circ u=x$.

Proof. Let $x=a+i b$ and $y=c+i d$ with $a, b \in C(\mathcal{J})$ and $c, d \in \mathcal{J}_{s a}$. Then

$$
\begin{aligned}
T_{x} T_{y} & =\left(T_{a}+i T_{b}\right)\left(T_{c}+i T_{d}\right)=T_{a} T_{c}+i T_{a} T_{d}+i T_{b} T_{c}-T_{b} T_{d} \\
& =T_{c} T_{a}+i T_{d} T_{a}+i T_{c} T_{b}-T_{d} T_{b}=T_{y} T_{x}
\end{aligned}
$$

as $a, b \in C(\mathcal{J})$ which proves (i).

(ii). Since $U_{y}=2 T_{y}^{2}-T_{y^{2}}$, we have

$T_{x} U_{y}=T_{x}\left(2 T_{y}^{2}-T_{y^{2}}\right)=2 T_{x} T_{y}^{2}-T_{x} T_{y^{2}}=\left(2 T_{y}^{2}-T_{y^{2}}\right) T_{x}=U_{y} T_{x}$ by part (i) (note that the associativity of $\mathcal{B}(\mathcal{J})$ is used here).

(iii). Since $x \in \mathcal{Z}(\mathcal{J}), x^{2} \in \mathcal{Z}(\mathcal{J})$ by Remark 3.2 . Hence by part (ii),

$$
\begin{aligned}
U_{x} U_{y} & =\left(2 T_{x}^{2}-T_{x^{2}}\right) U_{y}=2 T_{x}^{2} U_{y}-T_{x^{2}} U_{y} \\
& =2 U_{y} T_{x}^{2}-U_{y} T_{x^{2}}=U_{y} U_{x} .
\end{aligned}
$$

(iv). By part (i), $\left(x \circ u^{*}\right) \circ u=T_{u} T_{x} u^{*}=T_{x} T_{u} u^{*}=T_{x} e=x$. 
Theorem 3.4. Let $\mathcal{J}$ be a JB*-algebra with unit $e$ and let $b \in$ $\mathcal{Z}(\mathcal{J})$. Then for any unitary $u \in \mathcal{U}(\mathcal{J})$ and for any $x \in \mathcal{J}$ we have

(i) $\left(u^{*} \circ x\right) \circ u=u^{*} \circ(x \circ u)$;

(ii) $\left\{(b \circ u) u^{*} x\right\}=b \circ x$.

Proof. (i). If $\mathcal{J}$ is special then

$$
\begin{aligned}
\left(u^{*} \circ x\right) \circ u & =\frac{1}{4}\left(u\left(u^{*} x+x u^{*}\right)+\left(u^{*} x+x u^{*}\right) u\right) \\
& =\frac{1}{4}\left(2 x+u x u^{*}+u^{*} x u\right) \\
& =\frac{1}{4}\left(u^{*}(u x+x u)+(u x+x u) u^{*}\right)=u^{*} \circ(x \circ u) .
\end{aligned}
$$

Hence, by the Shirshov-Cohn theorem with inverses [5], we have in the general case $\left(u^{*} \circ x\right) \circ u=u^{*} \circ(x \circ u)$.

(ii). Since $b \in \mathcal{Z}(\mathcal{J})$ and $u \in \mathcal{U}(\mathcal{J})$, we get by Lemma 3.3 (iv) that

$$
(b \circ u) \circ u^{*}=b .
$$

Again by Lemma 3.3 (i),

$$
\left(u^{*} \circ x\right) \circ(b \circ u)=T_{\left(u^{*} \circ x\right)} T_{b} u=T_{b} T_{\left(u^{*} \circ x\right)} u=b \circ\left(u \circ\left(x \circ u^{*}\right)\right),
$$

and

$$
u^{*} \circ((b \circ u) \circ x)=T_{u^{*}} T_{x} T_{b} u=T_{b} T_{u^{*}} T_{x} u=b \circ\left(u^{*} \circ(x \circ u)\right),
$$

so by part (i)

$$
\left(u^{*} \circ x\right) \circ(b \circ u)=u^{*} \circ((b \circ u) \circ x) .
$$

Thus by (1) and (2),

$$
\begin{aligned}
\left\{(b \circ u) u^{*} x\right\} & =\left((b \circ u) \circ u^{*}\right) \circ x+\left(u^{*} \circ x\right) \circ(b \circ u)-((b \circ u) \circ x) \circ u^{*} \\
& =b \circ x .
\end{aligned}
$$

We now need a characterisation of the centre in terms of Hermitian operators. These are defined in terms of the numerical range of operators as follows (see [14], for example).

Definition 3.5. If $\mathcal{J}$ is a complex unital Banach Jordan algebra with unit $e$ and $D(\mathcal{J})=\left\{f \in \mathcal{J}^{*}: f(e)=\|f\|=1\right\}$ then, for $a \in \mathcal{J}$, the numerical range of $a$, denoted by $W(a)$, is defined by $W(a)=\{f(a): f \in D(\mathcal{J})\}$. The element $a$ is called Hermitian if $W(a) \subseteq \mathbb{R}$. The set of all Hermitian elements of $\mathcal{J}$ is denoted by Her $\mathcal{J}$. 
The Hermitian elements in a unital $J B^{*}$-algebra are exactly the self-adjoint elements (see [13]) but we shall need the following characterisation of the Hermitian operators on a $J B^{*}$-algebra, given in $[14]$.

Theorem 3.6. Let $\mathcal{J}$ be a $J B^{*}$-algebra with unit $e$. Then $S \in$ $\operatorname{Her} \mathcal{B}(\mathcal{J})$ if and only if $S=T_{a}+\delta$ where $\delta$ is $a^{*}$-derivation and $a=S(e)$ is self-adjoint.

We can now give a characterisation of the centre of a unitary isotope.

Theorem 3.7. Let $\mathcal{J}$ be a JB*-algebra with unit $e$ and let $u \in$ $\mathcal{U}(\mathcal{J})$. Let $\mathcal{A}$ be a $J C^{*}$-subalgebra of $\mathcal{B}(\mathcal{H})$ for some Hilbert space $\mathcal{H}$ with unit $e_{A}$ and let $w \in \mathcal{U}(\mathcal{A})$.

(i) If $x \in \mathcal{Z}(\mathcal{J})$ then $u \circ x \in \mathcal{Z}\left(\mathcal{J}^{[u]}\right)$.

(ii) If $a \in \mathcal{Z}\left(\mathcal{A}^{[w]}\right)$ then $\left(a \circ w^{*}\right) \circ w=a$.

(iii) If $z \in \mathcal{Z}\left(\mathcal{J}^{[u]}\right)$ then $u \circ\left(u^{*} \circ z\right)=z$.

(iv) Define $\psi: \mathcal{Z}(\mathcal{J}) \rightarrow \mathcal{Z}\left(\mathcal{J}^{[u]}\right)$ by $\psi(x)=u \circ x$. Then $\psi$ is an isometric ${ }^{*}$-isomorphism of $\mathcal{Z}(\mathcal{J})$ onto $\mathcal{Z}\left(\mathcal{J}^{[u]}\right)$.

Proof. (i). Let $x=a+i b$ where $a, b \in \mathcal{Z}(\mathcal{J})_{s a}$. Let $S=T_{a} \in$ $\operatorname{Her} \mathcal{B}(\mathcal{J})$. Then

$$
S(e)=T_{a}(e)=a \circ e=a \text { and } S(u)=u \circ a .
$$

As $S \in \operatorname{Her} \mathcal{B}(\mathcal{J}), S(u) \in\left(\mathcal{J}^{[u]}\right)_{s a}$ by Theorem 3.6. By Theorem 3.4 (ii),

$$
S(y)=T_{a}(y)=a \circ y=\left\{(a \circ u) u^{*} y\right\}=(a \circ u) \circ_{u} y
$$

for all $y \in \mathcal{J}$. Therefore, $S(y)=L_{S(u)}^{[u]}(y)$ for all $y \in \mathcal{J}$, where operator $L_{S(u)}^{[u]}$ stands for the multiplication by $S(u)$ in $\mathcal{J}^{[u]}$. Moreover, as $a \in \mathcal{Z}(\mathcal{J})$ we get by $\left[14\right.$, Theorem 14] that $S^{2} \in \operatorname{Her} \mathcal{B}(\mathcal{J})=$ $\operatorname{Her} \mathcal{B}\left(\mathcal{J}^{[u]}\right.$ ) because $\mathcal{B}\left(\mathcal{J}^{[u]}\right)=\mathcal{B}(\mathcal{J})$ (see Theorem 2.1). So again by $\left[14\right.$, Theorem 14], $S(u) \in \mathcal{Z}\left(\mathcal{J}^{[u]}\right)$ as $S=L_{S(u)}^{[u]}$. Therefore, $u \circ a \in \mathcal{Z}\left(\mathcal{J}^{[u]}\right)_{s a}$. Similarly, $u \circ b \in \mathcal{Z}\left(\mathcal{J}^{[u]}\right)_{s a}$. Hence $u \circ x=u \circ a+i u \circ b \in \mathcal{Z}\left(\mathcal{J}^{[u]}\right)$.

(ii). By Remark 3.2,

$$
\mathcal{Z}(\mathcal{A})=\{x \in \mathcal{A}: x y=y x\} .
$$


By Theorem 2.3, the isotope $\mathcal{A}^{[w]}$ is a $J C^{*}$-algebra and

$$
\mathcal{Z}\left(\mathcal{A}^{[w]}\right)=\left\{x \in \mathcal{A}: x w^{*} y=y w^{*} x\right\} .
$$

Now, if $a \in \mathcal{Z}\left(\mathcal{A}^{[w]}\right)$ then (by (4)) $a w^{*} y=y w^{*} a$ for all $y \in \mathcal{A}$. In particular,

$$
a w^{*}=w^{*} a .
$$

By part (i), $a \circ w^{*}=e_{A} \circ_{w} a \in \mathcal{Z}\left(\mathcal{A}^{[w]^{\left[e_{A}\right]}}\right)=\mathcal{Z}(\mathcal{A})$. So we have by (4) that

$$
\left(a \circ w^{*}\right) \circ w=\left(a \circ w^{*}\right) w=\frac{1}{2}\left(a w^{*}+w^{*} a\right) w
$$

hence by (5)

$$
\left(a \circ w^{*}\right) \circ w=\left(a w^{*}\right) w=a\left(w^{*} w\right)=a,
$$

as required .

(iii) Now, let $v$ be any unitary in $\mathcal{Z}\left(\mathcal{J}^{[u]}\right)$ (the centre of the unitary isotope $\mathcal{J}^{[u]}$ of the $J B^{*}$-algebra $\left.\mathcal{J}\right)$. Then $v$ is a unitary in $\mathcal{J}$ by Theorem 2.5. By [8, Corollary 1.14], $\mathcal{J}\left(e, u, u^{*}, v, v^{*}\right)$ is a $J C^{*}$ algebra and $v \in \mathcal{Z}\left(\left(\mathcal{J}\left(e, u, u^{*}, v, v^{*}\right)\right)^{[u]}\right)$. Hence, by (ii),

$$
u \circ\left(u^{*} \circ v\right)=v \text {. }
$$

If $z \in \mathcal{Z}\left(\mathcal{J}^{[u]}\right)$, then by the Russo-Dye Theorem (cf. [11]) for $C^{*}$ algebras there exist unitaries $v_{j} \in \mathcal{Z}\left(\mathcal{J}^{[u]}\right)$ and scalars $0 \leq \lambda_{j} \leq 1$ with $\sum_{j=1}^{n} \lambda_{j}=1$ for some $n \in \mathcal{N}$ such that $\frac{z}{\|z\|+1}=\sum_{j=1}^{n} \lambda_{j} v_{j}$ because $\left\|\frac{z}{\|z\|+1}\right\|<1$ (recall that $\mathcal{Z}\left(\mathcal{J}^{[u]}\right)$ is a $C^{*}$-algebra). Hence, by $(6)$,

$$
\begin{aligned}
u \circ\left(u^{*} \circ z\right) & =u \circ\left(u^{*} \circ(\|z\|+1) \sum_{j=1}^{n} \lambda_{j} v_{j}\right) \\
& =(\|z\|+1) \sum_{j=1}^{n} \lambda_{j}\left(u \circ\left(u^{*} \circ v_{j}\right)\right) \\
& =(\|z\|+1) \sum_{j=1}^{n} \lambda_{j} v_{j}=z .
\end{aligned}
$$

(iv). As $\psi=T_{u} \mid \mathcal{Z}(\mathcal{J}), \psi$ is linear and continuous by Lemma 1.2 (i). Let $z \in \mathcal{Z}\left(\mathcal{J}^{[u]}\right)$. Applying part (i) to $\mathcal{J}^{[u]}$ we get $e \circ_{u} z \in$ $\mathcal{Z}\left(\mathcal{J}^{[u]^{[e]}}\right)$. But $\mathcal{J}^{[u]^{[e]}}=\mathcal{J}$ by Lemma 2.4 and $e o_{u} z=\left\{e u^{*} z\right\}=$ $u^{*} \circ z$. Hence $u^{*} \circ z \in \mathcal{Z}(\mathcal{J})$. Moreover, $\psi\left(u^{*} \circ z\right)=u \circ\left(u^{*} \circ z\right)=z$ by part (iii). Thus $\psi$ maps $\mathcal{Z}(\mathcal{J})$ onto $\mathcal{Z}\left(\mathcal{J}^{[u]}\right)$. 
Further, $\|\psi(x)\| \leq\|u\|\|x\|$ while, by Lemmas 3.3 (i) and 1.2 (ii),

$$
\|x\|=\left\|T_{x} T_{u^{*}} u\right\|=\left\|T_{u^{*}} T_{x} u\right\| \leq\|x \circ u\|=\|\psi(x)\| .
$$

Thus $\psi$ is an isometry.

Finally, as $\psi(e)=u$ and $u$ is the unit of $\mathcal{J}^{[u]}$ it follows from $[12$, Theorem 6$]$ that $\psi$ is an isometric *-isomorphism.

Corollary 3.8. Let $\mathcal{J}$ be a unital $J B^{*}$-algebra. Then, for all $u, v \in$ $\mathcal{U}(\mathcal{J}), \mathcal{Z}\left(\mathcal{J}^{[u]}\right)$ is isometrically Jordan ${ }^{*}$-isomorphic to $\mathcal{Z}\left(\mathcal{J}^{[v]}\right)$.

Proof. By Theorem 2.5, $v \in \mathcal{U}(\mathcal{J})$. Hence, by Theorem 3.7, $\mathcal{Z}\left(\mathcal{J}^{[u]}\right)$ is isometrically ${ }^{*}$-isomorphic to $\mathcal{Z}\left(\mathcal{J}^{[u]^{[v]}}\right)$. However, by Corollary $2.6(\mathrm{i}), \mathcal{J}^{[u]^{[v]}}=\mathcal{J}^{[v]}$. This gives the required result.

An alternative proof of above Corollary 3.8 can be obtained by noting that $\mathcal{Z}\left(\mathcal{J}^{[u]}\right)$ is isometrically ${ }^{*}$-isommorphic to $\mathcal{Z}(\mathcal{J})$ and $\mathcal{Z}(\mathcal{J})$ is isometrically ${ }^{*}$-isomorphic to $\mathcal{Z}\left(\mathcal{J}^{[v]}\right)$ by Theorem 3.7 (applied twice). As the next example shows there need be no inclusion between the centre of a unital $J B^{*}$-algebra and the centre of its isotopes. In the following discussion $\mathcal{M}_{2}(\mathbb{C})$ denotes the standard complexification of the real Jordan algebra of all $2 \times 2$ symmetric matrices.

Example 3.9. If $u \in \mathcal{U}\left(\mathcal{M}_{2}(\mathbb{C})\right) \backslash \mathcal{Z}\left(\mathcal{M}_{2}(\mathbb{C})\right)$ then the unit $e \notin$ $\mathcal{Z}\left(\mathcal{M}_{2}(\mathbb{C})^{[u]}\right)$.

Indeed, $\mathcal{M}_{2}(\mathbb{C})^{[u]}$ is a 4 -dimensional $C^{*}$-algebra by Theorem 2.3 with 1-dimensional centre by the above Theorem 3.7. As $u$ does not belong to $\mathcal{Z}\left(\mathcal{M}_{2}(\mathbb{C})\right), u \notin S p(e)$ where $S p(e)$ denotes the linear span of $e$, and hence $e \notin S p(u)$. This gives that $e \notin \mathcal{Z}\left(\mathcal{M}_{2}(\mathbb{C})^{[u]}\right)$.

As a final point on the relationships between the centres it should be noted in the proof of Theorem 3.7 (i) that if $a \in \mathcal{Z}(\mathcal{J})$ and $S=$ $T_{a}$ then $S$ is left multiplication in any unitary isotope. In order to study the *-derivations it might be hoped that if $T \in \operatorname{Her} \mathcal{B}(\mathcal{J})$ then there exists a unitary isotope $\mathcal{J}^{[u]}$ such that $T$ is left multiplication operator in $\operatorname{Her} \mathcal{B}\left(\mathcal{J}^{[u]}\right)$ since as linear spaces $\mathcal{B}(\mathcal{J})=\mathcal{B}\left(\mathcal{J}^{[u]}\right)$ so $\operatorname{Her} \mathcal{B}(\mathcal{J})=\operatorname{Her} \mathcal{B}\left(\mathcal{J}^{[u]}\right)$. Unfortunately, this fails even when

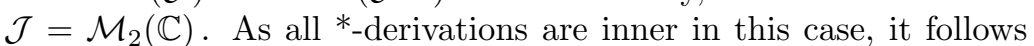
that $T \in \operatorname{Her} \mathcal{B}\left(\mathcal{M}_{2}(\mathbb{C})\right)$ if and only if $T=l_{a}+r_{b}$ where $a, b \in$ $\left(\mathcal{M}_{2}(\mathbb{C})\right)_{s a}$ and $l_{a}(x)=a x$ and $r_{b}(x)=x b$. 
Corollary 3.10. If $a, b \in \mathcal{M}_{2}(\mathbb{C})$ are given by $a=\left(\begin{array}{ll}1 & 0 \\ 0 & 2\end{array}\right)$, $b=\left(\begin{array}{cc}6 & 0 \\ 0 & 23\end{array}\right)$ and $T \in \operatorname{Her} \mathcal{B}\left(\mathcal{M}_{2}(\mathbb{C})\right)$ is defined by $T=l_{a}+r_{b}$, then $T$ is not left multiplication in any unitary isotope.

Proof. It was noted in Example 3.9 that if $u \in \mathcal{U}\left(\mathcal{M}_{2}(\mathbb{C})\right)$ then $\mathcal{M}_{2}(\mathbb{C})^{[u]}$ is a four-dimensional $C^{*}$-algebra with a one-dimensional centre so is isomorphic to $\mathcal{M}_{2}(\mathbb{C})$. By [4, Theorem 10], $\sigma(T)=$ $\sigma(a)+\sigma(b)=\{7,8,24,25\}$.

On the other hand, if $L_{c}^{[u]} \in \operatorname{Her} \mathcal{B}\left(\mathcal{M}_{2}(\mathbb{C})\right)$ with say $\sigma_{\mathcal{M}_{2}(\mathbb{C})}(c)=$ $\left\{\lambda_{1}, \lambda_{2}\right\}$ then $\sigma\left(L_{c}^{[u]}\right)=\left\{\lambda_{1}, \frac{\lambda_{1}+\lambda_{2}}{2}, \lambda_{2}\right\}$ again by [4, Theorem 10], so $\sigma\left(L_{c}^{[u]}\right)$ contains only three points. Hence $\sigma(T) \neq \sigma\left(L_{c}^{[u]}\right)$ for any unitary $u \in \mathcal{U}\left(\mathcal{M}_{2}(\mathbb{C})\right)$.

Acknowledgement. The author is indebted to Dr Martin A. Youngson for his help and encouragement during this work.

\section{REFERENCES}

[1] E. Alfsen, F. W. Shultz and E. Størmer, A Gelfand-Naimark theorem for Jordan algebras, Adv. in Math. 28 (1978), 11-56.

[2] C. V. DevaPakkiam, Jordan algebras with continuous inverse, Math. Jap. 16 (1971), 115-125.

[3] N. Jacobson, Structure and representations of Jordan algebras, Amer. Math. Soc., Providence, Rhode Island, 1968.

[4] G. Lumer and M. Rosenblum, Linear operator equations, Proc. Amer. Math. Soc. 10 (1959), 32-41.

[5] K. McCrimmon, Macdonald's theorem with inverses, Pacific J. Math. 21 (1967), 315-325.

[6] W. Rudin, Functional analysis, McGraw-Hill, New York, 1973.

[7] F. W. Shultz, On normed Jordan algebras which are Banach dual spaces, J. Funct. Anal. 31 (1979), 360-376.

[8] A. A. Siddiqui, Positivity of invertibles in unitary isotopes of JB*-algebras, submitted.

[9] H. Upmeier, Symmetric Banach manifolds and Jordan $C^{*}$-algebras, Amsterdam, 1985.

[10] J. D. M. Wright, Jordan $C^{*}$-algebras, Mich. Math. J. 24 (1977), 291-302.

[11] J. D. M. Wright and M. A. Youngson, A Russo-Dye theorem for Jordan $C^{*}$ algebras, Functional Analysis: Surveys and recent results (North Holland, 1977).

[12] J. D. M. Wright and M. A. Youngson, On isometries of Jordan algebras, J. London Math. Soc. 17 (1978), 339-344.

[13] M. A. Youngson, A Vidav theorem for Banach Jordan algebras, Math. Proc. Camb. Phil. Soc. 84 (1978), 263-272. 
[14] M. A. Youngson, Hermitian operators on Banach Jordan algebras, Proc. Edin. Math. Soc. (2nd ser.) 22 (1979), 169-180.

Akhlaq A. Siddiqui,

Department of Mathematics,

Prince Sultan University,

P. O. Box 66833,

Riyadh 11586, Saudi Arabia

saakhlaq@gmail.com

Received on 26 September 2006 and in revised form on 9 May $200 \%$. 